\title{
Statistical and Topological Atlas Based Brain Image Segmentation
}

\author{
Pierre-Louis Bazin and Dzung L. Pham \\ Johns Hopkins University, Baltimore, USA
}

\begin{abstract}
This paper presents a new atlas-based segmentation framework for the delineation of major regions in magnetic resonance brain images employing an atlas of the global topological structure as well as a statistical atlas of the regions of interest. A segmentation technique using fast marching methods and tissue classification is proposed that guarantees strict topological equivalence between the segmented image and the atlas. Experimental validation on simulated and real brain images shows that the method is accurate and robust.
\end{abstract}

\section{Introduction}

The segmentation of medical images into separate structures and organs often requires the incorporation of a priori information. The use of statistical atlases is one approach for encoding spatial and intensity information for each structure, and may include relational information as well [12]. In a typical scenario, the atlas is transformed to match the image of interest, and a statistical classification method combines the atlas and image information to segment the structures. An alternative is to perform a non-rigid registration of a single atlas to the image with the appropriate structures segmented by mapping the segmentation labels [3 4 5] . Hybrid methods incorporating statistical atlas and non-rigid registration have also been proposed [6]7].

In all cases, the atlas introduces a bias toward images most similar to the atlas images. In addition, many of the global and regional topological properties of the structures are ignored in these representations. For example, segmented structures may become disjointed or connect freely with neighboring structures, irrespective of the underlying anatomy. Several methods have been proposed to preserve topology in the case of a single object [14[15] or multiple, separate objects [168]. However, none of these methods is able to preserve the topology of groups of objects. Even if the topology of two objects is invariant, the topology of the union of these objects is not constrained and can be arbitrary (see Fig. 1). This is an important issue when segmenting multiple structures, since the true anatomy typically follows strict topological relationships.

In this paper, we show that topological constraints on both the structures and their groups can be used to encode continuity and relationships without biasing shape. We present a strictly homeomorphic atlas-based segmentation algorithm and apply it to the segmentation of the major structures of the brain in magnetic resonance (MR) images. Our method employs only a coarse statistical atlas of the shape, deriving the segmentation predominantly from the image and topological constraints. In the case of homogeneous structures, intensity prior distributions can be ignored, making the framework largely independent of the imaging modality.

N. Ayache, S. Ourselin, A. Maeder (Eds.): MICCAI 2007, Part I, LNCS 4791, pp. 94 101, 2007.

(C) Springer-Verlag Berlin Heidelberg 2007 

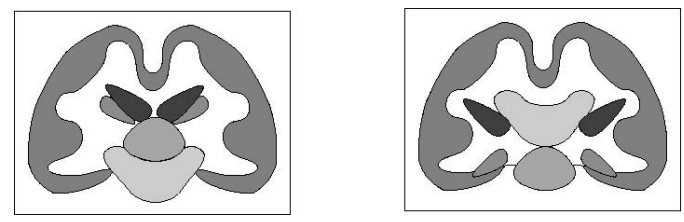

Fig. 1. Importance of the topology of groups: the individual structures in above images have the same topology, but the relationships between them are different, which corresponds to changes in the topology of groups (unions) of structures

The proposed algorithm significantly extends a topology-preserving tissue segmentation technique from our earlier work [8] by introducing several improvements. First, a statistical atlas and intensity relations are used to model objects with similar intensities. Second, a new topology preservation criterion is employed that maintains not only the topology of individual objects but also the topology of all groups of objects. With these improvements, the new approach is capable of segmenting more detailed structures, such as subcortical regions. Experiments on simulated and real images demonstrate the accuracy and robustness of the algorithm.

\section{Methods}

We seek to recover a set of $K$ anatomical structures from a MR image. Given a priori information about the structures shape and topology, we build an atlas to guide the segmentation. The atlas is first aligned with the image to segment, then a segmentation technique based on tissue classification and topology-preserving front propagation adapts the segmentation from the atlas to fit the image data.

\subsection{Statistical Atlas}

The first component of our algorithm is a statistical atlas, which will help distinguish structures with similar intensities. The atlas is built from a set of $N$ manual delineations of the structures of interest. For each image in the atlas, the delineation is rigidly aligned with the current atlas image, and a smooth approximation of the probabilities is accumulated. The smoothing replaces the step edge at the boundary of each structure in their binary delineation by a linear ramp over a band of size $\varepsilon$. The accumulated prior $p_{j k}$ represents the probability of being inside each structure as a function of the distance to its boundary and its variability over the atlas images, similarly to sigmoid-based representations [910]. By taking all these factors into account, we expect to reduce the bias toward the mean shape of the atlas and allow the statistical atlas to only influence areas most likely to be inside each structure. Fig. 2 shows the statistical priors computed from 18 subjects. Later in Section 3 it will be seen that the results of our method do not greatly depend on the size of $N$.

During the segmentation, the statistical atlas must be registered to the image. We use a joint segmentation and registration technique [11] that alternates between estimating 
the segmentation given the current atlas position, and then maximizes the following correlation energy with respect to $T$ given the current segmentation:

$$
E_{R}(T)=\sum_{j k} p_{T(j) k}^{q} u_{j k}^{q} .
$$

where $T$ is a rigid or affine transformation, $u_{j k}$ the membership function for structure $k$ at point $j$ defined in Section 2.3 and $q$ a fuzziness factor used in the underlying fuzzy segmentation method (see Sec.2.3. We use $q=2$ in this work. The transformation is computed using a gradient ascent technique. The initial alignment is computed using a Gaussian pyramid, while a single scale is sufficient to refine the alignment during the segmentation.

\subsection{Topological Atlas}

The second component of our algorithm is an atlas of the structures' topology, encoded in a parcellation of the brain. Anatomical structures can have a very complex geometry, yet they all tend to have a very simple topology, such as that of a sphere or torus. Groups of tissues and organs in contact provide additional topological properties. For instance, it is often assumed that the cerebral cortex has a spherical topology, but this requires to group together cerebral gray and white matter, sub-cortical structures and ventricles.

Creation of an appropriate topology atlas is non-trivial: manual segmentations have arbitrary topology, and inferring topological properties from anatomical atlases is not straightforward. As we are interested in the major structures of the brain, we created an atlas for the following structures, based on statistical atlases and anatomy textbooks: cerebral gray matter (CRG), cerebral white matter (CRW), cerebellar gray matter $(\mathrm{CBG})$, cerebellar white matter $(\mathrm{CBW})$, brainstem $(\mathrm{BS})$, ventricles (VEN), caudate (CAU), putamen (PUT), thalamus (THA) and sulcal cerebro-spinal fluid (CSF). Both the statistical and topology atlases are in the same coordinate space and are based on manual segmentations from the IBSR V2.0 dataset [12] with additional editing [8], as shown in Fig. 2

\subsection{Tissue Segmentation}

To perform the segmentation, we extend the FANTASM (Fuzzy And Noise Tolerant Adaptive Segmentation Method) [13] approach. Given the original image $I$, FANTASM minimizes an energy function with respect to the membership functions $u_{j k}$, the gain field $g_{j}$, and the class centroids $c_{k}$. The gain field is a smoothly varying function that we model as a low degree polynomial (see [11]).

As with any tissue classification technique, difficulties arise if adjacent structures have the same or very close centroid values; the boundary between them becomes more sensitive to noise, and the boundaries with other structures may be shifted away because of their lower membership values. The additional information provided by a statistical atlas will help remedy this issue but will also lower the influence of the signal intensity and blur the segmentation in areas of large variability between individuals. Because we model the topology of the structures to segment, we can also make use of the relationship between them to lower the influence of competing intensity clusters in regions that 


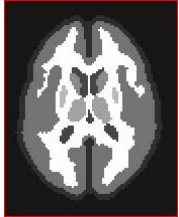

Topology

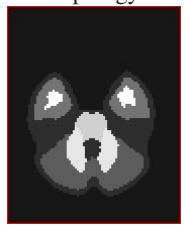

Topology

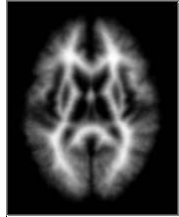

CRW (-2)

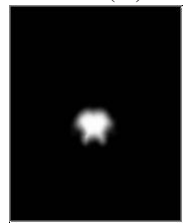

BS (0)

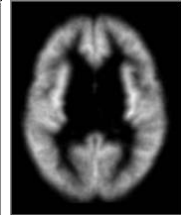

CRG (2)

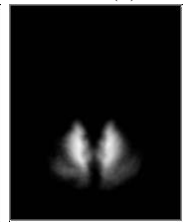

CBW (4)

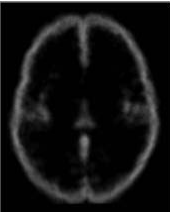

CSF (2)

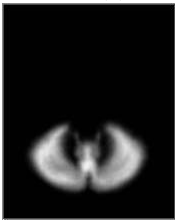

CBG (2)

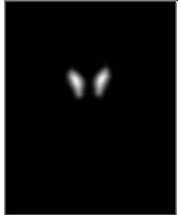

CAU (4)

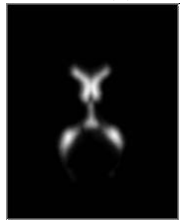

VEN (2)

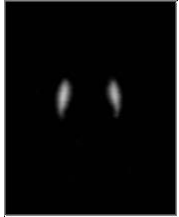

PUT (4)

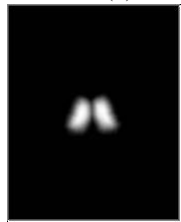

THA (2)

Fig. 2. The statistical and topological atlas: two slices from the topology atlas (left) and one from each structure prior (right). The Euler characteristic for each structure is given in parenthesis.

are spatially disconnected. We define global relationship sets $R_{G}(k)$ to be the structures in contact with $k$ according to the topology atlas, and local relationship sets $R_{L}(j)$ to be structures in contact at the boundary closest to the point $j$ in the current segmentation. The map $R_{L}(j)$ is obtained by searching for the structures in contact at all boundary points, and then propagating this information inside the volume (see example in Fig. 3 where each gray level represents a unique $R_{L}$ ).

We incorporate the atlas and relationship information into FANTASM, resulting in the following energy function:

$$
E_{S}=\sum_{j k} \frac{u_{j k}^{q}}{r_{j k}}\left\|g_{j} I_{j}-c_{k}\right\|^{2}+\frac{\beta}{2} \sum_{l \in N_{j}, m \neq k} \frac{u_{j k}^{q}}{r_{j k}} u_{l m}^{q},+\frac{\gamma}{2} \sum_{m \neq k} \frac{w_{k m}}{r_{j k}} u_{j k}^{q} p_{j m}^{q}
$$

The first term in (2) is the data driven term, the second term enforces smoothness on the memberships, and the third term controls the influence of the statistical atlas. The relationship function $r_{j k}$ is defined to be

$$
r_{j k}=\left\{\begin{array}{l}
1 k \in R_{L}(j) \\
\frac{1}{2} k \in R_{G}(l), l \in R_{L}(j) \\
0 \text { otherwise. }
\end{array}\right.
$$

The atlas weights $w_{k m}$ is defined as

$$
w_{k m}=\frac{s_{w} \max _{l n}\left|c_{l}-c_{n}\right|^{2}}{s_{w} \max _{l n}\left|c_{l}-c_{n}\right|^{2}+\left|c_{k}-c_{m}\right|^{2}}
$$

and is close to one when $c_{k} \simeq c_{m}$ but goes to zero when $c_{k} \neq c_{m}$. The relationship function penalizes against class configurations that are inconsistent with the topology atlas. For pixel classes that do not touch any classes near pixel $j, r_{j k}$ is set to zero, and the configuration possesses infinite energy. The atlas weights allow the priors to influence the segmentation only where the intensity contrast between structures is low. 


\subsection{Topology-Preserving Fast Marching Segmentation}

The energy function of (2) is used to compute membership functions for each structure in a fashion similar to FANTASM [13]. In addition, we compute a "hard" segmentation that is derived from the memberships but is constrained to be homeomorphic to the topology template.

We recently introduced the digital homeomorphism criterion that extends the binary case to multiple objects and guarantees that the atlas and segmented image are related by a homeomorphism in both the continuous and digital domain [17]. This improvement is essential as it allows a more truthful representation of the anatomy than previous methods.

The segmentation itself is performed using two successive iterations of a fast marching front propagation technique, first thinning the structures into a skeleton-like object and then growing the structures back to find the optimal boundary, using a minimal path strategy [18]8]. The speed function of the fast marching is a function of the memberships:

$$
\begin{aligned}
& f^{-}=(1-\alpha) u_{j k}+\alpha \kappa_{j k} \\
& f^{+}=(1-\alpha)\left(1-u_{j k}\right)+\alpha\left(1-\kappa_{j k}\right)
\end{aligned}
$$

where $\kappa_{j k}$ is a measure of the curvature of the boundary for structure $k$ at $j$ normalized in $[0,1]$ and $\alpha$ a weighting parameter. $f^{-}$is used in the thinning algorithm, $f^{+}$ in the growing algorithm. The thinning is stopped when the volume remaining inside each structure is below a given fraction of the original volume (in this work, $1 / 3$ of the volume). The digital homeomorphism criterion is checked at all steps, ensuring that the topology of all structures and groups are preserved at all times.

The complete algorithm is as follows:

1. Align atlases to image and set initial segmentation to the topological atlas.

2. Build the local relationship map $R_{L}$ from the current segmentation.

3. Compute the memberships $u_{j k}$, centroids $c_{k}$ and the inhomogeneity field $g_{j}$.

4. Thin structures using the fast marching algorithm with $f^{-}$.

5. Grow back the structures using $f^{+}$and update the segmentation.

6. Refine the alignment of the atlases.

7. Loop to step 2 until convergence.

The convergence criterion is the relative amount of change in the energy $E$ per iteration, which typically becomes lower than $10^{-3}$ in 10 to 20 iterations in the following experiments (approximatively 45 to 60 minutes). The overall complexity of the algorithm is $O(N \log N)+O(K N)$, with $N$ the size of the image and $K$ the number of structures. Parameters were determined empirically and fixed for all experiments.

\section{Experiments and Validation}

\subsection{Simulated Images}

A first set of experiments was conducted with the Brainweb phantom [19]: several levels of noise and field inhomogeneity were simulated for T1 and T2-weighted modalities and the corresponding images segmented. Because the original Brainweb ground 
truth is only concerned with the three major tissue classes (gray matter, white matter, cerebro-spinal fluid), we performed a manual segmentation to separate cerebellum/brainstem, cerebrum, and grouped sub-cortical structures. The results from the segmentation were grouped accordingly (CAU, PUT and THA as sub-cortical structures SUB, BS and CBW as CBS), and the difference measured with the Dice overlap measure $\left(D(A, B)=\frac{2 A \cap B}{A+B}\right)$, see Table 1 Euler characteristic were found identical for all segmentations, as expected.

Table 1. Dice coefficients for the Brainweb dataset, for varying noise (N) and inhomogeneity (I)

\begin{tabular}{l|l|lll|llllll} 
& & \multicolumn{7}{|c|}{ Tissues } & \multicolumn{7}{c}{ Structures } \\
Modality & \multicolumn{1}{|c|}{ N / I } & WM & GM & CSF & CRW & CRG & CBS & CBG & SUB & VEN \\
\hline T1 & N 0\%, I 0\% & 0.953 & 0.935 & 0.884 & 0.963 & 0.941 & 0.767 & 0.895 & 0.850 & 0.897 \\
& N 1\%, I 0\% & 0.951 & 0.934 & 0.884 & 0.961 & 0.940 & 0.766 & 0.896 & 0.849 & 0.897 \\
& N 3\%, I 0\% & 0.947 & 0.928 & 0.882 & 0.957 & 0.934 & 0.762 & 0.892 & 0.845 & 0.896 \\
& N 3\%, I 20\% & 0.947 & 0.927 & 0.880 & 0.957 & 0.933 & 0.761 & 0.896 & 0.846 & 0.896 \\
& N 3\%, I 40\% & 0.947 & 0.918 & 0.880 & 0.956 & 0.931 & 0.765 & 0.898 & 0.842 & 0.898 \\
& N 5\%, I 0\% & 0.938 & 0.919 & 0.876 & 0.948 & 0.923 & 0.755 & 0.888 & 0.836 & 0.896 \\
& N 5\%, I 20\% & 0.938 & 0.919 & 0.877 & 0.948 & 0.923 & 0.754 & 0.891 & 0.839 & 0.896 \\
& N 5\%, I 40\% & 0.939 & 0.919 & 0.876 & 0.949 & 0.922 & 0.754 & 0.893 & 0.838 & 0.896 \\
& N 7\%, I 0\% & 0.927 & 0.906 & 0.869 & 0.937 & 0.909 & 0.745 & 0.884 & 0.828 & 0.894 \\
& N 9\%, I 0\% & 0.916 & 0.895 & 0.862 & 0.926 & 0.897 & 0.737 & 0.877 & 0.820 & 0.893 \\
\hline T1 & Mean & 0.940 & 0.921 & 0.877 & 0.950 & 0.925 & 0.756 & 0.891 & 0.839 & 0.896 \\
& St.Dev. & 0.007 & 0.013 & 0.012 & 0.011 & 0.014 & 0.010 & 0.006 & 0.010 & 0.001 \\
\hline T2 & Mean & 0.902 & 0.887 & 0.879 & 0.912 & 0.891 & 0.724 & 0.871 & 0.797 & 0.856 \\
& St.Dev. & 0.019 & 0.020 & 0.007 & 0.020 & 0.023 & 0.013 & 0.009 & 0.020 & 0.014 \\
\hline Euler characteristic & -2 & 6 & 2 & -2 & 2 & 0 & 2 & 6 & 2
\end{tabular}

The Brainweb 'ground truth' has arbitrary topology and our segmentation will always deviate from it because of the topological constraints it follows (the Euler characteristics for the Brainweb WM,GM and CSF tissue classes are 6, -1750 and -174 respectively). Despite this, the segmentation is very close to the Brainweb ground truth almost everywhere. The larger difference in CBS is due to the mean intensity of the brainstem, which is between the WM and GM intensities considered in Brainweb, and thus is not segmented as a homogeneous region in the ground truth. The topology enforces continuity for each structure, resulting in a segmentation very robust to high noise levels. The segmentation results for T1 and T2 images are similar, despite the signal differences between the modalities.

\subsection{Real Images}

We tested the algorithm on the IBSR dataset, where we could compare each label individually (see Fig. 3). Because we used the same dataset to create the atlas, we performed four different evaluations. In the first, all 18 images from the dataset were used to create the atlas, whereas the second, third and fourth atlases respectively used the first 8,3 and 1 images of the dataset. We also compared the results with the segmentation obtained by performing a non-rigid registration with HAMMER [4] and transferring the object labels. The overlap with the original segmentation is computed as before (see Table 2).

The sulcal CSF is grouped with CRG and CBG in the IBSR segmentation, thus the overlap with the original ground truth are lowered for CRG and CBG and irrelevant for CSF. The algorithm recovers all structures with an accuracy significantly higher than 
Table 2. Dice coefficients for the IBSR dataset

\begin{tabular}{ll|lllllllll} 
& & \multicolumn{1}{|c}{ Structures } \\
& & CRW & CRG & BS & CBW & CBG & CAU & PUT & THA & VEN \\
\hline Atlas & Mean & 0.887 & 0.816 & 0.847 & 0.846 & 0.840 & 0.797 & 0.761 & 0.775 & 0.832 \\
$01-18$ & St.Dev. & 0.012 & 0.049 & 0.016 & 0.026 & 0.030 & 0.037 & 0.030 & 0.054 & 0.048 \\
\hline Atlas & Mean & 0.886 & 0.816 & 0.848 & 0.843 & 0.845 & 0.798 & 0.755 & 0.765 & 0.832 \\
$01-08$ & St.Dev. & 0.012 & 0.048 & 0.019 & 0.027 & 0.028 & 0.039 & 0.034 & 0.056 & 0.049 \\
\hline Atlas & Mean & 0.883 & 0.826 & 0.843 & 0.846 & 0.848 & 0.799 & 0.743 & 0.763 & 0.831 \\
$01-03$ & St.Dev. & 0.015 & 0.041 & 0.022 & 0.026 & 0.028 & 0.040 & 0.043 & 0.052 & 0.051 \\
\hline Atlas & Mean & 0.879 & 0.832 & 0.853 & 0.839 & 0.859 & 0.794 & 0.744 & 0.760 & 0.833 \\
01 & St.Dev. & 0.018 & 0.038 & 0.016 & 0.038 & 0.024 & 0.043 & 0.044 & 0.051 & 0.051 \\
\hline HAMMER & Mean & 0.788 & 0.782 & 0.744 & 0.763 & 0.848 & 0.740 & 0.599 & 0.773 & 0.662 \\
& St.Dev. & 0.032 & 0.038 & 0.059 & 0.042 & 0.032 & 0.039 & 0.071 & 0.046 & 0.078
\end{tabular}
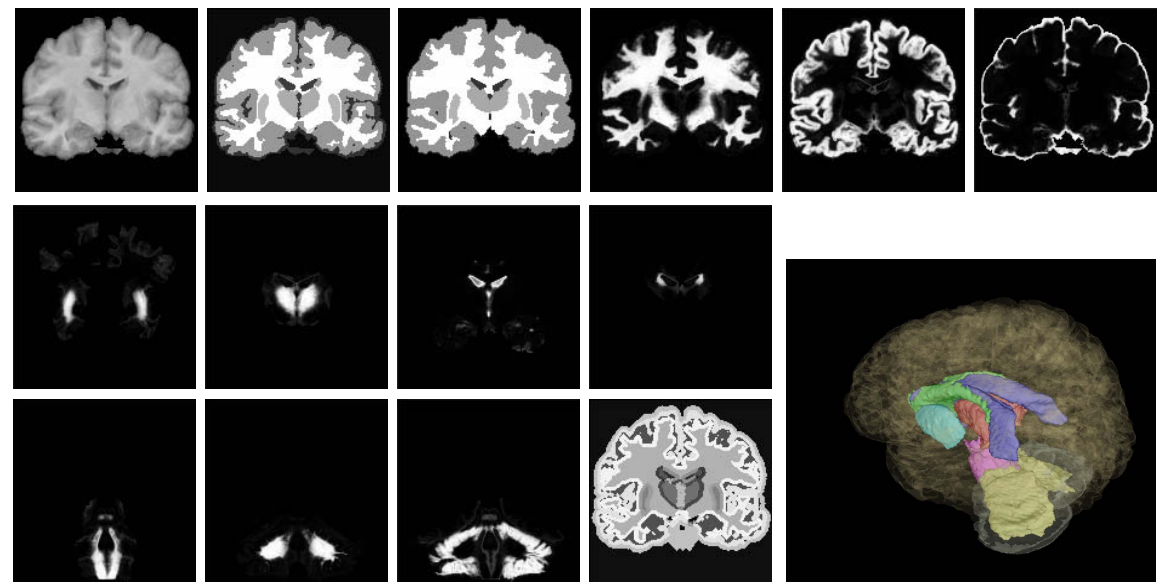

Fig. 3. Example of segmentation from the IBSR dataset. From left to right, top to bottom: original image, computed segmentation, manual segmentation, membership functions for CRW, CRG, CSF, PUT, THA, VEN, CAU, BS, CBW, CBG, relation map $R_{L}, 3 \mathrm{D}$ rendering.

the non-rigid registration approach and similar to the reported inter-rater scores of [2]. The overlap is somewhat lower for the sub-cortical structures, due to their smaller size, non-constant intensities, and inaccurate boundaries. The results are mostly independent of the number of images used to generate the atlas, indicating that precise prior information about shape and location is not required by the algorithm.

\section{Conclusion}

In this paper, we presented a new framework for the segmentation of multiple structures in MR images. By combining topology constraints with smooth atlas priors, we are able to recover the main structures of the brain, both cortical and sub-cortical. The proposed algorithm is modality-independent, robust to high levels of noise and inhomogeneity, and the influence of spatial priors is limited, reducing the bias inherent to atlas-based methods. More importantly, the framework guarantees a strict homeomorphism between all groups of structures in the atlas and the segmented images, allowing 
an accurate representation of the anatomy that may be readily used for cortical unfolding and diffeomorphic shape analysis applications.

\section{References}

1. Leemput, K.V., Maes, F., Vandermeulen, D., Suetens, P.: Automated model-based tissue classification of MR images of the brain. IEEE Trans. Medical Imaging 18(10), 897-908 (1999)

2. Fischl, B., Salat, D.H., Busa, E., Albert, M., Dieterich, M., Haselgrove, C., van der Kouwe, A., Killiany, R., Kennedy, D., Klaveness, S., Montillo, A., Makris, N., Rosen, B., Dale, A.M.: Whole brain segmentation: Automated labeling of neuroanatomical structures in the human brain. Neuron 33, 341-355 (2002)

3. Christensen, G.E., Joshi, S.C., Miller, M.I.: Volumetric transformation of brain anatomy. IEEE Trans. Medical Imaging 16(6), 864-877 (1997)

4. Shen, D., Davatzikos, C.: Hammer: Hierarchical attribute matching mechanism for elastic registration. IEEE Trans. Medical Imaging 21(11) (2002)

5. Rohde, G.K., Aldroubi, A., Dawant, B.M.: The adaptative bases algorithm for intensity-based nonrigid image registration. IEEE Trans. Medical Imaging 22(11), 1470-1479 (2003)

6. Ciofolo, C., Barillot, C.: Brain segmentation with competitive level sets and fuzzy control. In: Proc. Int. Conf. Information Processing in Medical Imaging, Glenwood Springs (2005)

7. Pohl, K.M., Fisher, J., Levitt, J.J., Shenton, M.E., Kikins, R., Grimson, W.E.L., Wells, W.M.: A unifying approach to registration, segmentation and intensity correction. In: Proc. Int. Conf. Medical Image Computing and Computer-Assisted Intervention, Palm Springs (2005)

8. Bazin, P.L., Pham, D.: Topology-preserving tissue classification of magnetic resonance brain images. IEEE Trans. Medical Imaging 26(4), Special Issue on Computational Neuroanatomy (2007)

9. Rousson, M., Xu, C.: A general framework for image segmentation using ordered spatial dependency. In: Proc. Int. Conf. Medical Image Computing and Computer-Assisted Intervention, Copenhagen (2006)

10. Pohl, K.M., Fisher, J., Shenton, M.E., McCarley, R.W., Grimson, W.E.L., Kikins, R., Wells, W.M.: Logarithm odds maps for shape representation. In: Proc. Int. Conf. Medical Image Computing and Computer-Assisted Intervention, Copenhagen (2006)

11. Pham, D., Bazin, P.L.: Simultaneous registration and tissue classification using clustering algorithms. In: Proc. Int. Symposium on Biomedical Imaging, Arlington (2006)

12. Worth, A.: Internet brain segmentation repository (1996), http: //www.cma.mgh.harvard.edu/ibsr/

13. Pham, D.L.: Spatial models for fuzzy clustering. Computer Vision and Image Understanding 84, 285-297 (2001)

14. Malandain, G., Bertrand, G., Ayache, N.: Topological segmentation of discrete surfaces. Int. J. Computer Vision 10(2), 183-197 (1993)

15. Han, X., Xu, C., Prince, J.L.: A topology preserving level set method for geometric deformable models. IEEE Trans. Pattern Analysis and Machine Intelligence 25(6), 755-768 (2003)

16. Mangin, J.F., Frouin, V., Bloch, I., Regis, J., Lopez-Krahe, J.: From 3d magnetic resonance images to structural representations of the cortex topography using topology preserving deformations. J. Mathematical Imaging and Vision 5, 297-318 (1995)

17. Bazin, P.L., Ellingsen, L., Pham, D.: Digital homeomorphisms in deformable registration. In: Proc. Int. Conf. Information Processing in Medical Imaging, Kerkrade (2007)

18. Li, H., Yezzi, A., Cohen, L.: 3d brain segmentation using dual-front active contours with optional user interaction. Int. J. Biomedical Imaging, 1-17 (2006)

19. Collins, D.L., Zijdenbos, A.P., Kollokian, V., Sled, J.G., Kabani, N., Holmes, C., Evans, A.: Design and construction of a realistic digital brain phantom. IEEE Trans. Medical Imaging 17(3) (1998) 